\title{
Pre-Operative and Intra-Operative Factors for Conversion of Laparoscopic Cholecystectomy to Open Choleystectomy
}

Dr. Mohammad Abu Nasar ${ }^{1}$, Dr. Ramendranath Talukdar ${ }^{2 *}$, Dr. Kuldeep Raj ${ }^{3}$, Dr. P. N. Agarwal ${ }^{4}$, Dr. Aditya ${ }^{1}$, Dr. Sowbir Some ${ }^{1}$, Dr. Uma Pradhan ${ }^{1}$, Dr. Ravi Kumar ${ }^{1}$

\footnotetext{
${ }^{1}$ Post Graduate student, Department of General Surgery, SGT Medical College \& Hospital \& Research Institute, Badli Rd, near Sultanpur Bird Sanctuary, Budhera, Gurugram, Haryana 122505, India

${ }^{2}$ Associate Professor, Department of General Surgery, SGT Medical College \& Hospital \& Research Institute, Badli Rd, near Sultanpur Bird Sanctuary, Budhera, Gurugram, Haryana 122505, India

${ }^{3}$ Professor, Department of General Surgery, SGT Medical College \& Hospital \& Research Institute, Badli Rd, near Sultanpur Bird Sanctuary, Budhera, Gurugram, Haryana 122505, India

${ }^{4}$ Head and Professor, Department of General Surgery, SGT Medical College \& Hospital \& Research Institute, Badli Rd, near Sultanpur Bird Sanctuary, Budhera, Gurugram, Haryana 122505, India
}

DOI: $10.36347 / \mathrm{sasjs} .2021 . \mathrm{v} 07 \mathrm{i} 02.011$

| Received: 21.01.2021 | Accepted: 06.02.2021 | Published: 16.02.2021

*Corresponding author: Dr. Ramendranath Talukdar

Abstract Original Research Article

Laparoscopic cholecystectomy is the gold standard treatment for gall stone disease. The advantages of laparoscopy over open has been documented in multiple studies with an increase patient acceptability due to less pain and early return to work. However, certain factors have been documented in predicting difficult laparoscopic cholecystectomy. In our institution, which is a tertiary care center in a rural community, we conducted a study on 280 consecutive patients fitting the inclusion criteria to determine the various pre-operative and intra-operative factors for conversion to open cholecystectomy from standard 4 port laparoscopic cholecystectomy. We observed a conversion rate of $6.05 \%$ with dense adhesion being the major cause of conversion. Various conversion rate have been reported in literatures ranging from $1.6 \%-19 \%[1,2]$. Other causes for conversion in the 17 patients being frozen Calots, Mirizzi's syndrome, slippage of ligaclip, pre-operative factors like ERCP and $4.5 \mathrm{~mm}$ thickness of the gallbladder.

Keywords: Laparoscopic Cholecystectomy, Open Cholecystectomy, Dense adhesion, Calot's triangle, Open conversion, Gallbladder.

Copyright $(92021$ The Author(s): This is an open-access article distributed under the terms of the Creative Commons Attribution 4.0 International License (CC BY-NC 4.0) which permits unrestricted use, distribution, and reproduction in any medium for non-commercial use provided the original author and source are credited.

\section{INTRODUCTION}

Laparoscopic cholecystectomy is a gold standard treatment of gall stone disease. It is minimal invasive surgical procedure in general surgery and has replaced the invasive procedure of open cholecystectomy in the treatment of gall stone disease. Gall stone disease is a major health problem more in female as compare to male. Its prevalence rate varies in different population [3, 4]. Abdominal pain is the common symptom in presence of gall stone. The first open cholecystectomy was done by Carl Langenbach of Germany (1846-1901) and first laparoscopic cholecystectomy was done by Professor Erich of Germany (1882). The procedure of laparoscopy is performed by multiple skilled general surgeon and laparoscopic surgeons. Numerous studies show laparoscopic cholecystectomy is more common than open cholecystectomy because short hospital stay, early hospital discharge and less surgical stress [5]. The conversion also depends on surgeon experience and quality of instrument. The conversion rate is different in different areas of world. We wanted to know the conversion rate of elective cases of gall stone in our hospital.

\section{MATERIAL AND METHODS}

The prospective observational study was conducted from December 2018 to December 2019 in Department of General Surgery, SGT Medical College, Hospital and Research Institute on 280 consecutive patients diagnosed with gall stone disease. Patients with an age between 18 to 70 years without any symptom of acute cholecystitis, or signs of jaundice and diagnosis of common bile duct calculi or hepatobiliary carcinoma were included in this study. Upon admission, routine pre-operative investigations were carried out along with transabdominal ultrasound abdomen and pre-anesthetic checkup. Standard 4 port laparoscopic cholecystectomy was performed by experienced general surgeon and laparoscopic surgeons of different units. 


\section{RESULTS}

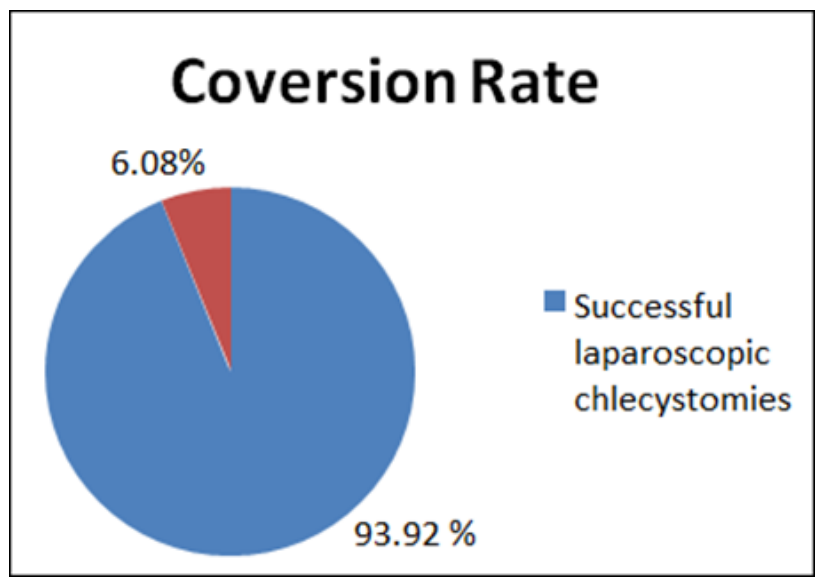

Fig-1: Conversion Rate

The conversion rate in our study of 280 patients was $6.05 \% .17$ patients were converted to open cholecystectomy. The main reason for conversion being dense adhesion, difficult anatomy of Calot's (inability to identify the structure over the Calots triangle), CBD injury and cystic artery Liga clip slippage. Gender distribution of patients were female $246(87.85 \%)$ and male $34(12.15 \%)$.

Further analysis revealed that 72 patients were in age group of 18-30 years of age, 73 patients in age group 31-40, 59 patients between $41-50$ age, 50 patients between 5160 years of age and 26 patients between 6170 years of age. The female to male ratio being 7.2:1.

\section{Gender Distribution}

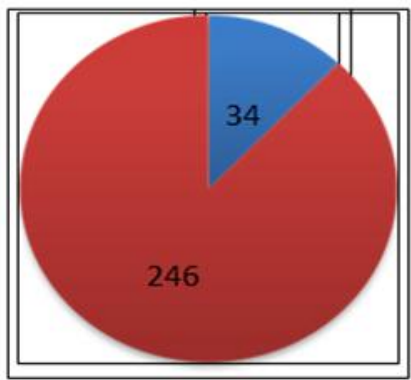

$\square$ Male

$\square$ Female

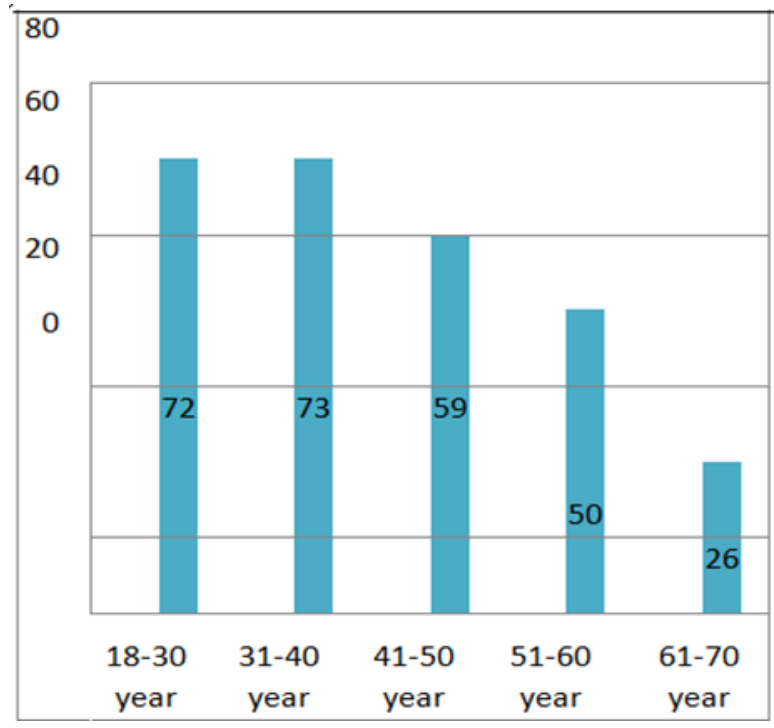

Fig-3: Age Distribution

The mean age of both sexes was 42.34 years of age. All patients were taken for laparoscopic cholecystectomy under the protocol of general anesthesia, out of which $17(6.07 \%)$ were converted into open cholecystectomy and in 263 patient $(93.93 \%)$ laparoscopic procedures was successful. Among 17 converted patients, 6 patients $(35.29 \%)$ were male, 11 patients $(64.71 \%)$ were female (Fig-4). Analysis regarding the reason of conversion to open procedure in total 17 converted patients was dense adhesion in 9 patients, 2 patients had difficult anatomy (inability to identify the calot's triangle), 2 patients had cystic duct injury due to short cystic duct, 2 patients had conversion due to impacted stone at the neck, 1 patient of Mirizzi syndrome had conversion due to CBD injury and 1 patient had conversion due to cystic artery clip slippage.

Fig-2: Gender Distribution 


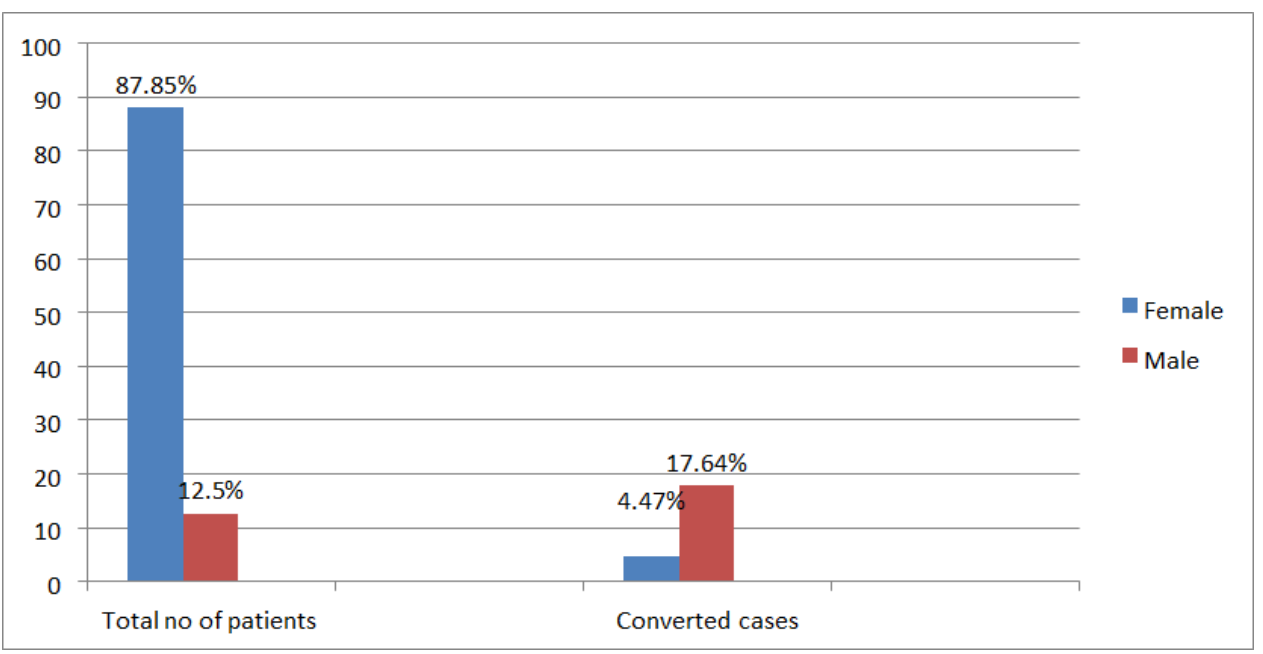

Fig-4: Percentage of gender distribution in total number of patients with gallbladder stones and percentage of conversion rate in male and female

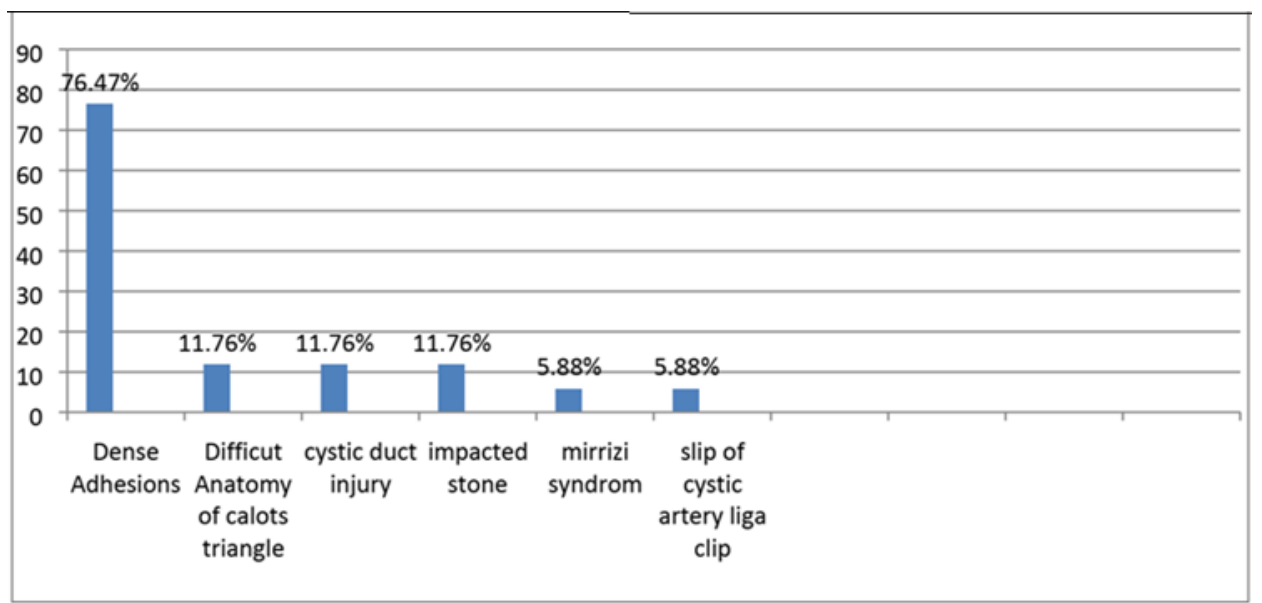

Fig 5: Percentage of various factors in conversion

\section{DISCUSSION}

Gall stone disease is a major health problem world-wide and females are more prone to gallstone disease as compared to males. In our study of 280 patients, $87.85 \%$ of the study population were female and $12.14 \%$ were males with an average age of 47 years. This result coincides with that of Frazee et al., [6] For reasons unknown, some asymptomatic cases progress to symptomatic stage due to obstruction of the cystic duct by gallstone. Symptomatic gallstones can complicate leading to gall stone pancreatitis, formation of an empyema or mucocele or can perforate with fistula formation. Complicated gallstone disease develops in 3-5\% of symptomatic patients per year. Over a period of 20 years, about $2 / 3$ of asymptomatic patients with gallstone remains symptom free [7]. To avoid the complications, patients are advised for surgery. Laparoscopic cholecystectomy is the treatment of choice. With a shorter duration of hospital stay, less post-operative pain, decreased wound complications and early return to normal activity, it has replaced open cholecystectomy.
Conversion of laparoscopic cholecystectomy to open cholecystectomy is sometimes required in difficult cholecystectomy to prevent complications, ensuring the safety of the patients.

In our prospective study of 280 patients, $4.47 \%$ of the total number of female patients $(n=246)$ was converted to open and $17.64 \%$ of the male patients $(n=34)$ were converted to open cholecystectomy. This was similar to Brodsky et al., [8] who also found the male gender as a significant determinant for conversion to open cholecystectomy than the females. The reason for the higher conversion rates in male patients remains unexplained. In our study, we have observed the male patients with intense inflammation and fibrosis, resulting in a more difficult dissection of the Calot's triangle which is consistent with the study by Lim et al., [9].

There were 206 patients in our study below the age of 50 with $3.43 \%$ of them were converted to open procedure. Among the 76 patients, with average age more than 50 years, $14.28 \%$ of them were converted to 
open cholecystectomy. Our research coincides with the one done by Frazee et al., [10].

Gallbladder wall thickness has been cited as a major predictor for conversion from laparoscopic to open procedure in many western literatures. In our study, 10 patients with an average wall thickness of $4.5 \mathrm{~mm}$ on ultrasound, 2 of them were converted to open. In another study by Tayeb $\mathrm{M}$ et al., [11] 58\% of the patients with thickness of the gallbladder wall more than $3 \mathrm{~mm}$ were converted from laparoscopic to open cholecystectomy, suggesting that gall bladder thickness is a good predictive factor for conversion to open surgery. Our finding coincides with these studies.

According to our study, one of the most common factors in a difficult laparoscopic cholecystectomy or the necessity to convert to open cholecystectomy was the presence of dense adhesions in the Calot's triangle. Presence of adhesions could lead to injury of the cystic artery, CBD or cause a tear in the gall bladder causing leakage of bile and stones. Out of 17 converted patients $4 / 6$ male $(66.66 \%)$ and $5 / 11$ female $(45.45 \%)$ patients, were showing dense adhesion during the procedure requiring conversion to open.

The other reasons for conversions from laparoscopic to open include difficult and unclear anatomy of Calot's triangle (inability to identify the structure over the Calot's triangle), cystic duct injury, Mirizzi's Syndrome and cystic artery liga clip slippage. $2(33.33 \%)$ among them were converted to open, 2 patients $(33.33 \%)$ were converted to open due to inability to identify the structures in the Calot's triangle. One study by Le VH, Smith DE, Johnson BL [12], 22\% among 3341 patients required conversion to open cholecystectomy due to difficult anatomy, showing poor positive correlation.

Pre-operatively 20 patients were shown to have impacted stone at the neck on USG reports out of them 2 patients $(10.00 \%)$ were converted to open cholecystectomy because of intra-operative finding of adhesion with impacted stone at the neck.

Other factors associated with conversion are:

- $2(0.70 \%)$ patients were converted to open due to Frozen Calot's triangle with short cystic duct which got injured during dissection.

- $1(0.35 \%)$ patient diagnosed as Mirrizi syndrome was converted to open and had CBD injury during dissection. One study by Memon MR [13] in which total patients were 1224 shows conversion due to CBD injury in $1(0.08 \%)$, Mirizzi syndrome in 1 $(0.08 \%)$.

- Slippage of ligaclip from cystic artery, leading to severe bleeding, resulted in 1 patient being converted to open. This is consistent with the data by Kaushik R [14].

- 2 patients had undergone ERCP preoperatively for
Choledocholithiasis, in which $1(50.00 \%)$ patient got converted to open due to dense adhesions. A study by $M$ Abdel Dayem et al., [15] in total patients of 531 patients in which preoperative ERCP was done with $\mathrm{p}$ value of 0.027 .

- 4 patients were investigated with MRCP for dilated common bile duct. $2(50.00 \%)$ patients got converted to open cholecystectomy due to dense adhesions and short cystic duct $(\mathrm{p}=1.00)$.

Overall, most common causes for conversion was dense adhesion which was more common in female as compared to male followed by inability to finding the structure over the Calot triangle, short cystic duct injury, common bile duct injury and slippage of cystic artery clip. In our study the result of conversion was comparatively less as compared to others studies by Simopoulos C et al., [16], Sanabria JR et al., [17].

\section{CONCLUSION}

An appreciation of these factors would predict the conversion that will allow appropriate planning by the surgeon. Of the 280 patients in whom laparoscopic cholecystectomy was attempted, $17(6.07 \%)$ required conversion to open surgery. The most common reason for conversion was dense adhesions around the Calot's triangle.

Laparoscopic cholecystectomy is a gold standard treatment of gall bladder stone disease. Significant predictors of difficult cholecystectomy include male patients, thickened gallbladder wall on ultrasound, dense adhesions from fundus to Calot's triangle, frozen Calot's, unclear anatomy and iatrogenic injury to ducts and vessels. These factors are more of importance which leads to conversion from laparoscopic to open cholecystectomy. It is important to keep in mind that conversion from laparoscopic surgery to open surgery is not seen as a complication, but rather a matter of sound surgical judgment as safety of the patient is of foremost importance.

\section{DECLARATION \\ Conflict of Interest: None}

\section{Funding: None}

\section{REFERENCE}

1. Randhawa SR, Tanverr I, Zafar H, Rashid A. Frequency of conversion to open cholecystectomy among male and female patients undergoing laparoscopic cholecystectomy. JUMDC. 2014;5(1):42-6.

2. Jan H, Rafiq MS, Khan A, Wazir HK, Saeed T. An audit of laparoscopic cholecystectomy; J Med Sci, 2015; 23(1):46-49.

3. Awan NA, Hamid F, Mir IN, Ahmad MM, Shah AA, Asimi A, et al. Factors resulting in conversion of laparoscopic cholecystectomy to open cholecystectomy-institution based study. 
International Surjery Journal. 2017;5(1):132-7.

4. Javaid A, Bashir T, Ali MM. Laparoscopic cholecystectomy; Conversion rate, Experience of single surgeon over 4-year period. J Surg Surgical Res. 2017;3(2):030-3.

5. Ghnnam W, Malek J, Shebl E, Elbeshry T, Ibrahim A. Rate of conversion and complications of laparoscopic cholecystectomy in a tertiary care centre in Saudi Arabia. Annals of Saudi Medicine.2010;55(2):103-9.

6. Frazee RC, Thames T, Appel M, Roberts J, Symmonds R, Snyder S, Hendricks J, Smith R, Allen TW. Laparoscopic cholecystectomy: a multicenter study. Journal of Laparoendoscopic Surgery. 1991 Jun;1(3):157-9.

7. Attili AF, De Santis A, Capri R, et al. The natural history of gallstones: the GREPCO experience. The GREPCO Group. Hepatology. 1995;21:655.

8. Brodsky, M Tayeb, Syed Ahsan Raza, MR Khan, R Azami. Department of Surgery, Aga Khan University, Karachi, Pakistan.

9. Lim et al (Lim SH, Salleh I, Poh BK, Tay KH. Laparoscopic cholecystectomy: an audit of our training programme. ANZ journal of surgery. 2005 Apr;75(4):231-3.

10. Frazee RC, Thames T, Appel M, Roberts J, Symmonds R, Snyder S, Hendricks J, Smith R, Allen TW. Laparoscopic cholecystectomy: a multicenter study. Journal of Laparoendoscopic Surgery. 1991 Jun;1(3):157-9.

11. Tayeb M, Raza SA, Khan MR, Azami R. Conversion from laparoscopic to open cholecystectomy: multivariate analysis of preoperative risk factors. Journal of postgraduate medicine. 2005;51(1):17.]

12. Le VH, Smith DE, Johnson BL. Conversion of laparoscopic to open cholecystectomy in the current era of laparoscopic surgery. Am Surg. 2012 Dec; 78(12): 1392-5.

13. Memon MR, Muhammad G, Arshad S, Jat MA, Bozdar AG, Shah SQ. Study of open conversion in laparoscopic cholecystectomy. Gomal J Med Sci. 2011;9(1):51-54

14. Kaushik R. Bleeding complications in laparoscopic cholecystectomy: Incidence, mechanisms, prevention and management. Journal of minimal access surgery. $2010 \mathrm{Jul} ; 6(3): 59$.

15. AbdelDayem M, Osgood L, Escofet X. A New Preoperative Scoring System to Predict Difficulty of Laparoscopic Cholecystectomy and Risk of Conversion to Open Surgery. Indian J Surg, 2020;82:501-506.

16. Simopoulos C, Botaitis S, Polychronidis A, Tripsianis G, Karayiannakis AJ. Risk factors for conversion of laparoscopic cholecystectomy to open cholecystectomy. Surgical Endoscopy and Other Interventional Techniques. 2005 Jul 1;19(7):905-9.

17. Sanabria JR, Gallinger S, Croxford R, Strasberg SM. Risk factors in elective laparoscopic cholecystectomy for conversion to open cholecystectomy. Journal of the American College of Surgeons. 1994 Dec;179(6):696-704. 